\title{
Stiff Fluid Cosmological Models in Five Dimensional Space-Time Based On Lyra Geometry
}

\author{
Kalpana Pawar ${ }^{1}$, Rishikumar Agrawal ${ }^{2}$ \\ 1. Department of Mathematics, Shivaji Science College, Nagpur, India \\ 2. Department of Mathematics, Hislop College, Nagpur, India
}

\begin{abstract}
In this paper we have constructed stiff fluid cosmological models by considering five dimensional Kaluza-Klein space-time based on Lyra geometry in the frame work of scalar tensor theory of gravitation proposed by Saez and Ballester, which are obtained for two different cases: constant displacement vector and time dependent displacement vector. Also some physical and kinametical properties of the models are discussed.
\end{abstract}

Key words: Five dimensional cosmological models, stiff fluid, Lyra geometry.

\section{Introduction}

In recent years many efforts have been taken to construct alternative theories of gravitation. Einstein's idea of geometrizing gravitation in general theory of relativity motivated others to geometrize other physical things. Weyl (1918) proposed a modification of Riemannian manifold in order to unify gravitation and electromagnetism, but due to the non integrability of length transfer this theory was never considered seriously. Lyra (1951) proposed a modification of Riemannian geometry in which he introduced a gauge function to remove the one integrability of length of a vector under parallel transport. In this theory both the scalar and tensor fields have intrinsic geometrical significance.

The scalar-tensor theories of gravitation proposed by Brans and Dicke (1961), Nordtvedt (1970), Ross (1972) and Dunn (1974), Saez and Ballester (1985) have formulated a scalar-tensor theory of gravitation in which the metric is coupled with a dimensionless scalar field in a simple manner. This theory also suggests a possible way to solve missing matter problem in non-flat FRW cosmologies. Various five dimensional cosmological models in Lyra manifold are constructed by Rahaman (2002 - 03) and Singh (2004), Reddy (200507), Mohanty (2007-08).

The study of higher dimensional cosmological models is motivated mainly by the possibility of geometrically unifying the fundamental interactions of the universe. In the context of the Kaluza-Klein and super string theories higher dimensions have, recently, acquired much significance. Also, the higher dimensional theory is important at the early stages of the evolution of the universe Appleqluist et al. (1987). Rahaman et al. (2002), Chatterjee (1993) and Khadekar et al. (2005) have investigated higher dimensional string cosmological models in general relativity. In particular, Reddy (2003a, 2006) and Reddy et al. (2006, 2007) have discussed some string cosmological models in Saez-Ballester scalar-tensor theory of gravitation in five dimensions.

In this paper we have considered a five dimensional Kaluza-Klein metric with stiff fluid distribution in Lyra geometry. We have reproduced the exact cosmological models for two cases: constant displacement vector and time dependent displacement vector. We have also discussed some physical and kinematical properties of the Models.

\section{Metric and Field Equations}

Consider the five dimensional Kaluza-Klein metric in the form,

$\mathrm{ds}^{2}=\mathrm{dt}^{2}-\mathrm{R}^{2}\left(\mathrm{dx}^{2}+\mathrm{dy}^{2}+\mathrm{dz}^{2}\right)-\mathrm{A}^{2} \mathrm{dm}^{2}$,

where $\mathrm{A}$ and $\mathrm{R}$ are functions of cosmic time $\mathrm{t}$ only and the fifth coordinate is taken to be space-like. The field equations in normal gauge for Lyra's manifold as proposed by Sen (1957) and Sen and Dunn (1971) are given by,

$\mathrm{R}_{\mathrm{ij}}-\frac{1}{2} \mathrm{~g}_{\mathrm{ij}} \mathrm{R}+\frac{3}{2} \phi_{\mathrm{i}} \phi_{\mathrm{j}}-\frac{3}{4} \mathrm{~g}_{\mathrm{ij}} \phi_{\mathrm{k}} \phi^{\mathrm{k}}=-\chi \mathrm{T}_{\mathrm{ij}}$

Where $\phi_{\mathrm{k}}$ is the displacement vector given by,

$\phi_{\mathrm{k}}=(\beta, 0,0,0,0)$

The energy momentum tensor,

$\mathrm{T}_{\mathrm{ij}}=(\mathrm{p}+\rho) \mathrm{u}_{\mathrm{i}} \mathrm{u}_{\mathrm{j}}-\mathrm{pg}_{\mathrm{ij}}$

together with the co-moving coordinates

$\mathrm{g}_{\mathrm{ij}} \mathrm{u}^{\mathrm{i}} \mathrm{u}^{\mathrm{j}}=1$ 
Here $p, \rho$, and $\mathrm{u}^{\mathrm{i}}$ are isotropic pressure, energy density and five velocity vector of the cosmic fluid distribution respectively.

The field equations (2) together with (3), (4), and (5) for the space-time metric (1) yield the following equations, $3\left(\frac{\dot{\mathrm{R}}^{2}}{\mathrm{R}^{2}}+\frac{\dot{\mathrm{R}} \dot{\mathrm{A}}}{\mathrm{RA}}\right)-\frac{3 \beta^{2}}{4}=\chi \rho$

$2 \frac{\ddot{R}}{\mathrm{R}}+\frac{\dot{\mathrm{R}}^{2}}{\mathrm{R}^{2}}+2 \frac{\dot{\mathrm{R}} \dot{\mathrm{A}}}{\mathrm{RA}}+\frac{\ddot{\mathrm{A}}}{\mathrm{A}}+\frac{3 \beta^{2}}{4}=-\chi \mathrm{p}$

$3 \frac{\ddot{R}}{\mathrm{R}}+3 \frac{\dot{\mathrm{R}}^{2}}{\mathrm{R}^{2}}+\frac{3 \beta^{2}}{4}=-\chi \mathrm{p}$

where overhead dot denotes differentiation with respect to $t$.

\section{Cosmological Models}

In this section we derive exact solutions of the field equations (6) to (8) for stiff fluid equation i.e. for $p=\rho$.

Here there are four unknowns R, A, $\beta$; and p involved in three field equations (6) to (8). In order to derive explicit solutions, we consider the following cases:

Case (I): $\beta=$ constant

In this case, on solving (6) to (8) for $p=\rho$, we obtained

$3 \frac{\dot{R}}{\mathrm{R}}=-\frac{\ddot{A}}{\mathrm{~A}}=\mathrm{k}$ (say).

where $\mathrm{k}$ is an arbitrary constant.

$\mathrm{A}=\frac{\mathrm{e}^{-\mathrm{kt}+\mathrm{k} 1}}{\mathrm{k}}$

and $\mathrm{R}=\mathrm{e}^{\frac{\mathrm{kt}}{3}+\mathrm{k}_{3}}$

where $\mathrm{k}_{3}$ is the constant of integration.

Using in equation (8), we get $p=-\frac{c}{x}$,

where, $\mathrm{c}=\frac{2 \mathrm{k}^{2}}{3}+\frac{3 \beta^{2}}{4}$

Hence, metric (1) assumes the form

$\mathrm{ds}^{2}=\mathrm{dt}^{2}-\mathrm{e}^{2 \frac{\mathrm{kt}}{3}+\mathrm{k}_{3}}\left(\mathrm{dx}^{2}+\mathrm{dy}^{2}+\mathrm{dz}^{2}\right)-\frac{\mathrm{e}^{-2 \mathrm{kt}+2 \mathrm{k} 1}}{\mathrm{k}^{2}} \mathrm{dm}^{2}$.

Scalar expansion :

$\theta=\mathrm{u}_{\mathrm{i} i}^{\mathrm{i}}=0$.

Case (II): $\beta=\beta(\mathrm{t})$

In this case, we consider an analogue of power law,

i.e. $\mathrm{A}=\mathrm{aR}$,

(where a is an arbitrary constant) to obtain four unknowns $\mathrm{R}, \mathrm{A}, \mathrm{p}$ and $\beta$ involved in field equations (6) to (8) and to find the corresponding metric.

Using (14) in (6) to (8), we get

$6 \frac{\dot{\mathrm{R}}^{2}}{\mathrm{R}^{2}}-\frac{3 \beta^{2}}{4}=\chi \rho$

$3 \frac{\ddot{\mathrm{R}}}{\mathrm{R}}+3 \frac{\dot{\mathrm{R}}^{2}}{\mathrm{R}^{2}}+\frac{3 \beta^{2}}{4}=-\chi \rho$

Solving (15) and (16) using $p=\rho$, we obtained

$\mathrm{R}=\left[4\left(\mathrm{bt}+\mathrm{b}_{1}\right)\right]^{\frac{1}{4}}$

Equation (14) $\Rightarrow$

$A=a\left[4\left(b t+b_{1}\right)\right]^{\frac{1}{4}}$

Substituting value of $\mathrm{R}$ in (15), we get

$\chi \rho=\frac{6 b^{2}}{\left[4\left(b t+b_{1}\right)\right]^{2}}-\frac{3 \beta^{2}}{4}$

It is impossible to obtain separate value of $\rho$ and $\beta$.

We consider, $\beta=\frac{1}{\mathrm{~b}_{2} \mathrm{t}+\mathrm{b}_{3}}$

Therefore $\chi \rho=\frac{6 b^{2}}{\left[4\left(b t+b_{1}\right)\right]^{2}}-\frac{3}{4} \frac{1}{\left(b t+b_{1}\right)^{2}}$

In this case, the line element (1) becomes

$$
\mathrm{ds}^{2}=\mathrm{dt}^{2}-\left[4\left(\mathrm{bt}+\mathrm{b}_{1}\right)\right]^{\frac{1}{2}}\left(\mathrm{dx}^{2}+\mathrm{dy}^{2}+\mathrm{dz}^{2}\right)-\mathrm{a}^{2}\left[4\left(\mathrm{bt}+\mathrm{b}_{1}\right)\right]^{\frac{1}{2}} \mathrm{dm}^{2}, \quad(22)
$$


Scalar expansion :

$\theta=\mathrm{u}_{; \mathrm{i}}^{\mathrm{i}}=\frac{\mathrm{b}}{\mathrm{bt}+\mathrm{b}_{1}}$.

\section{Conclusion}

We have derived the exact solutions of the field equations for the two cases i.e., $\beta=$ constant and $\beta=\beta(t)$.

In case (I), equation (13) shows that at initial epoch $t=0$, the metric becomes flat. As time increases the three space coordinates expands while the fifth coordinate i.e. the extra dimension contracts. At infinite time the extra dimension becomes unobservable. The scalar of expansion $\mu$ in this case is zero. This behaviour is similar to that of the cosmic string model obtained by Reddy in four dimensional Lyra manifold.

In case (II), equation (22) indicates that the line element is flat at the initial epoch $t=0$ while the space coordinates expands with the increase in time. The scalar of expansion of $\theta$ in this case is

$$
\theta=\frac{\mathrm{b}}{\mathrm{bt}+\mathrm{b}_{1}}
$$

This model does not

approach isotropy for large values of $t$.

\section{Acknowledgement}

Authors are grateful to Dr. G. D. Rathod, Dr. R.V. Saraykar and Dr. G. S. Khadekar for their constant encouragement and fruitful discussion.

\section{References}

[1]. Alvarez, E., BelenGavela, M.: Phys. Rev. Lett., 51, 931 (1983)

[2]. Chodos, A., Detweiler, S.: Phys. Rev. D., 21, 2167 (1980)

[3]. Salam, A., Strathdee, J.: Ann. Phys. 141, 316 (1982)

[4]. Schwarz, J.: Nucl. Phys. B, 226, 269 (1983)

[5]. Wely, H.: Sber Preuss. Acad. Wiss. Berlin, 465, (1918)

[6]. Halford, W.D.: Aust. J. Phys., 23, 863 (1970)

[7]. Rahaman, F., Chakraborty, S., Begum, N., Hossain, M., Kalam, M.: FIZIKA B, 11, 57 (2002)

[8]. Rahaman, F., Chakraborty, S., Das, S., Hossain, M., Bera, J.: Pramana J. Phys. 60, 453 (2003)

[9]. Rahaman, F., Das, S., Begum, N., Hossain, M.: Pramana J. Phys. 61, 153 (2003)

[10]. Reddy, D.R.K.: Astrophys. Space Sci. 286, 365 (2003a)

[11]. Reddy, D.R.K.: Astrophys. Space Sci. 286, 397 (2003b)

[12]. Reddy, D.R.K.: Astrophys. Space Sci. 300, 381 (2005)

[13]. Reddy, D.R.K.: Astrophys. Space Sci. 305, 139 (2006)

[14]. Reddy, D.R.K., Rao, M.V.S.: Astrophys. Space Sci. 302, 157 (2006)

[15]. Reddy, D.R.K., Venkateshwara Rao, N.: Astrophys. Space Sci. 277, 461 (2001)

[16]. Reddy, D.R.K., Naidu, R.L., Rao, V.U.M.: Astrophys. Space Sci. 306, 185 (2006)

[17]. Singh, G.P., Deshpande, R.V., Singh, T.: Pramana J. Phys. 63, 937 (2004)

[18]. Mohanty, G., Mahanta, K.L., Sahoo, R.R.: Astrophys. Space Sci., 306, 269 (2007)

[19]. Mohanty, G., Mahanta, K.L., Bishi, B.K.: Astrophys. Space Sci., 310, 273 (2007)

[20]. Mohanty, G., Mahanta, K.L.: Astrophys. Space Sci., 312, 301 (2007)

[21]. Mohanty, G., Mahanta, K.L.: Turk J. Phys., 32, 299-303 (2008)

[22]. Sen, D.K.: Z. Phys. 149, 311 (1957)

[23]. Sen, D.K., Dunn, K.A.: J. Math. Phys., 12, 578 (1971) 